Reprod. Nutr. Dévelop., 1980, 20 (3 B), 747-758.

\title{
Comportement maternel et relations mères-jeunes chez les araignées
}

par B. KRAFFT, A. HOREL

Laboratoire de Biologie du Comportement Case Officielle 140, 54037 Nancy Cedex

Summary. Maternal behaviour and mother-young relationships in spiders.

Most species of spiders leave their egg-sacs in a sheltered place. However, some of them look after or carry their egg-sacs until the spiderlings hatch. Others extend their maternal behaviour to feeding their young by regurgitation or by the distribution of prey.

The adaptative aspect of this maternal behaviour is shown by a reduced rate of reproduction in the species whose care of progeny is the most sophisticated.

The maternal care of the egg-sac depends on the physiological state of the female, that state, in turn, being maintained by the presence of the egg-sac. The tolerance exhibited by the mother towards her offspring implies at least two mechanisms : (i) inhibition of predatory behaviour in response to the movements of an offspring at a distance, (ii) inhibition of cannibalism when in confact with an offspring. The parental relationship can be complemented by cooperation between the young that involves a tolerance mechanism probably close to that of social spiders.

\section{Introduction .}

De nombreuses espèces d'Araignées abandonnent leur cocon immédiatement après la ponte. La femelle se contente d'entourer ses œufs de plusieurs couches protectrices de soie et choisit un emplacement plus ou moins abrité, favorable à l'incubation des œufs et au développement des jeunes. Les espèces sédentaires vivant dans un abri permanent surveillent leur cocon et peuvent éventuellement s'occuper de leurs jeunes. Les Araignées vagabondes ont adopté deux types de comportement différents. Les unes transportent leur cocon dans leurs chélicères ou attaché aux filières, les autres modifient leur mode de vie et deviennent sédentaires pendant le développement de leurs œufs et de leurs jeunes.

Le développement du comportement maternel implique l'apparition d'interactions complexes empêchant la femelle de consommer ses œufs ou ses jeunes et permettant chez certaines espèces une coordination des activités entre la mère et sa progéniture. II existe peu d'études concernant la dynamique du comportement maternel chez les Araignées et elles sont anciennes. Ces travaux ont cependant montré qu'il existait des mécanismes de régulation entraînant une certaine plasticité du comportement maternel (Bonnet 1940 ; Palmgren, 1944), à une époque où on insistait sur l'aspect stéréotypé des comportements. 
Le comportement maternel esł probablement à l'origine des sociétés d'Araignées. Les jeunes Araignées nourries par la mère restent souvent groupées après la mort de la femelle et coopèrent en manifestant un comportement comparable à celui des espèces sociales.

\section{Les soins au cocon et aux jeunes.}

a) Soins au cocon.

La première forme de comportement maternel correspond à une surveillance du cocon pendant quelques jours, pouvant se continuer, suivant les espèces, jusqu'au moment de l'éclosion des jeunes. Agelena labyrinthica (Agelenidae) meurt en automne et ses jeunes sortent du cocon au printemps. Dans la même famille, Coelofes ferrestris garde son cocon dans son terrier jusqu'à l'éclosion des jeunes (Tretzel, 1961). Le même comportement s'observe chez les Amaurobiidae (Cloudsley-Thompson, 1955). Les Mygales terricoles (Nemesia caementaria et Cteniza moggridgei) surveillent leur cocon jusqu'à la sortie des jeunes (Buchli, 1960, 1968). Le comportement maternel est très souvent lié à la possession d'un terrier ou d'un abri permanent. Il est très largement répandu chez les Theridiidae (Bristowe, 1958 ; Hirschberg, 1969 ; Locket, 1926) et s'observe également chez certaines Salticides du genre Lyssomanes (Eberhard, 1974). Les Pholcides, certains Loxoscelides (Loxosceles reclusa) (Enders, 1976 ; Hite ef al., 1966), les Pisaurides (Bonnet, 1940 ; Le Pape, 1972) et les Lycosides (Dietlein, 1967 ; Higashi et Rovner, 1975 ; Job, 1974 ; Meyer, 1928) transportent leur cocon.

La surveillance du cocon par la femelle peut être complétée par des soins favorisant le développement des jeunes. Dolomedes plantarius (Pisauridae) distend progressivement les parois du cocon à mesure du développement des jeunes et l'humidifie (Bonnet, 1940). Six jours avant la sortie des jeunes, Peucetia viridans (Oxyopidae) découpe une ouverture latérale dans son cocon suspendu et l'incline de 40 à $80^{\circ}$, ce qui amène cette ouverture à la face inférieure (Randall, 1977). Elargissement et ouverture du cocon sont fréquents chez de nombreuses espèces, en particulier chez les Lycosides (Eason et Whitcomb, 1965 ; Higashi et Rovner, 1975), chez certains Theridiides (Hirschberg, 1969) ef chez Stegodyphus sarasinorum (Jacson ef Joseph, 1973). Les Lycosides transportent leur cocon accroché à leurs filières et l'exposent au soleil lorsque la température est basse. Elles se tiennent à l'ombre lorsque l'ensoleillement est trop intense. Ce transport du cocon s'observe même chez les Lycosides sédentaires telles que Sosyppus floridanus (Brach, 1976) et Geolycosa godeffroyi (Humphreys, 1974).

Les ssins au cocon sont de règle chez les Araignées sociales. Anelosimus eximius transporte son cocon ef le fixe temporairement aux fils de la toile (Brach, 1975). Les individus d'Agelena consociata suspendent leur cocon dans des chambres aménagées à l'intérieur des niches. Lorsqu'on détache ces cocons et qu'on les dépose sur la toile de chasse ou sur le plancher des galéries de la niche, les Araignées les raccrochent.

b) Soins aux jeunes.

Les soins au cocon peuvent se prolonger par des soins aux jeunes. Pisaura mirabilis suspend son cocon dans une pouponnière peu avant la sortie des jeunes et reste à 
proximité sans pour autant les nourrir. Lyssomanes sp. (Salticidae) garde ses jeunes pendant deux semaines (Eberhard, 1974). L'Araignée vagabonde Clubiona kulezynski (Clubionidae) s'enferme dans une cellule de soie, $y$ élabore son cocon et surveille ses jeunes pendant une à deux semaines sans prendre de nourriture (Buckle, 1971). Après la ponte, la femelle adopte temporairement une vie sédentaire. Ce changement d'activité n'apparaît pas chez les Lycosides puisqu'elles transportent leurs jeunes sur le dos pendant une ou deux semaines (Dietlein, 1967 ; Eason et Whitcomb, 1965 ; Fujii, 1976 ; Job, 1974). Elles ne les nourrissent pas mais s'arrêtent parfois à proximité de source d'eau pour leur permettre de s'abreuver.

La nutrition des jeunes s'observe chez la Mygale Nemesia caementaria (Buchli, 1960), Sosyppus floridanus (Brach, 1976), Loxosceles reclusa (Hite et al., 1966), Coelotes terrestris (Tretzel, 1961), Agelena consociata (Krafft, 1970) et certaines Amaurobiidae. La mère capture des proies ef les abandonne aux jeunes après les avoir injectées de sucs digestifs. Ce mode de nutrition peut être complété ou remplacé par une régurgitation de substances nutritives par la femelle, comme ce fut observé chez les Theridiides ef les Eresides (Bristowe, 1958 ; Hirschberg, 1969 ; Kullmann, 1972 ; Locket, 1926). Le liquide nourricier distribué par la mère ne correspond pas à une simple régurgitation de la nourriture absorbée. Il provient d'une autolyse des tissus qui épuise rapidement la femelle ef semble responsable de sa mort (Kullmann et Zimmermann, 1974). Dans ces deux familles, la nutrition des jeunes par régurgitation peut s'accompagner d'une prolongation de la vie grégaire ef d'une coopération entre les jeunes pour la construction du nid et la capture des proies, même après la mort de la mère. On observe là une évolution du comportement familial vers le comportement social (Krafft, 1979 ; Shear, 1970).

Les jeunes de Theridion tepidariorum ne sont pas nourris par la mère et se dispersent immédiatement après l'éclosion (Hirschberg, 1969). Ceux de Theridion pictum et Theridion saxatile (Achaearanea riparia) sont nourris à l'aide de proies tuées par la femelle (Hirschberg, 1969 ; Norgaard, 1956). Theridion impressum nourrit simultanément ses jeunes avec des proies et par régurgitation dès leur sortie du cocon. Après la première mue les jeunes dévorent la femelle et se dispersent sans manifester de coopération pour la capture des proies (Kullmann, 1970-71, 1972 ; Kullmann et Zimmermann, 1974). Theridion sysiphium nourrit exclusivement ses jeunes par régurgitation jusqu'à la première mue. Leur alimentation est complétée ensuite par des proies. La mère est rarement dévorée par les jeunes. Ceux-ci coopèrent dans la capture des proies même en l'absence de la femelle, jusqu'à la quatrième mue (Kullmann, 1970-71, 1972 ; Kullmann, Sittertz et Zimmermann, 1971 ; Kullmann et Zimmermann, 1974). Anelosimus studiosus est une Araignée subsociale dont la mère nourrit ses jeunes par trophallaxie et en leur distribuant des proies. Elle meurt lorsque les jeunes ont effectué la cinquième mue et se dispersent au stade subadulte (Brach, 1977 ; Kullmann et Zimmermann, 1974). Anelosimus eximius est une espèce sociale.

Parmi les Eresides, Stegodyphus pacificus nourrit ses jeunes par régurgitation jusqu'à la deuxième mue, mais ne leur distribue pas de proie. Le cadavre de la femelle est alors dévoré par les jeunes. Ceux-ci restent cependant groupés jusqu'à la cinquième mue ef coopèrent. L'alimentation est mixte et la dispersion intervient après la cinquième mue chez Stegodyphus lineatus. Les jeunes de l'Araignée terricole Eresus niger sont nourris par trophallaxie jusqu'à la deuxième mue, puis mangent la femelle et 
coopèrent jusqu'à la sixième mue. Stegodyphus sarasinorum et Stegodyphus mimosarum manifestent un comportement maternel du même type et sont sociales (Bradoo, 1972 ; Jacson et Joseph, 1973 ; Kullmann, 1970-71, 1972 ; Kullmann, Sittertz et Zimmermann, 1971 ; Kullmann, Nawabi et Zimmermann, 1972 ; Kullmann ef Zimmermann, 1974 ; Wickler 1973).

\section{Aspects adaptatifs du comportement maternel.}

\section{a) Développement du cocon.}

II est possible, mais pas démontré, que la surveillance ou le transport du cocon par la femelle assure une protection des œufs contre les prédateurs et les parasites. Ces derniers sont particulièrement nombreux (Eason et al., 1967). Clubiona kulezynskii ne fuit pas lorsqu'elle garde son cocon, même en réponse aux interventions de l'expérimentateur (Buckle, 1971). Lyssomanes sp. défendrait ses œufs et ses jeunes (Eberhard, 1974). Lubin (1974) a remarqué un comportement de défense contre les parasites chez l'Araignée sociale territoriale Cyrtophora moluccencis. Lorsqu'un Hyménoptère parasite vole à travers la colonie à la recherche d'un cocon, certaines femelles s'agrippent à leur cocon et le secouent vigoureusement. Ce comportement agit comme un signal, provoquant une réponse analogue chez les autres Araignées. Cependant Pardosa, qui pourtant transporte son cocon, ne réagit pas lorsqu'un Hyménoptère parasite vient y déposer ses œufs (Kessler et Fokkinga, 1973).

L'ouverture ef l'inclinaison du cocon semblent jouer un rôle important chez Peucefia viridans, car en l'absence de soins maternels, les jeunes sortent trois à quatre jours plus tard et manifestent un cannibalisme réduisant leur nombre (Randall, 1977). L'élargissement du cocon procure un espace plus important aux jeunes qui effectuent une ou plusieurs mues à l'intérieur de l'enveloppe (Fujii, 1978). Son ouverture par la femelle est indispensable aux Lycosides ef en particulier à Lycosa punctulata dont les jeunes ne peuvent s'extraire du cocon par leur propres moyens (Eason et Whitcomb, 1965).

Par leur comportement, les Lycosides effectuent une thermorégulaiion du cocon. En se plaçant alternativement à l'ombre et au soleil, Geolycosa godeffroyi maintient la température de son cocon à un niveau à peu près constant et supérieur à la température ambiante lorsqu'il fait frais (Humphreys, 1974). Theridion saxatile abrite son cocon sous une cloche constituée de grains de sable agglomérés. Exposé en plein soleil, la température de cet abri peut atteindre $45^{\circ} \mathrm{C}$, or les jeunes meurent si ils sont exposés $4 \mathrm{~min}$ à une température de $44^{\circ} \mathrm{C}$. Lorsque la température s'élève la femelle décroche son cocon et le place à l'ombre. Des expériences faites en étuve ont montré que la femelle sort le cocon de l'abri lorsque la température dépasse $36^{\circ} \mathrm{C}$. En dessous de $30^{\circ} \mathrm{C}$, il est remis sous la cloche (Norgaard, 1956). II n'est pas douteux que ces diverses formes de régulation thermique favorisent le développement des embryons.

b) Développement des jeunes.

Les jeunes bénéficient de la même protection contre les prédateurs et les parasites que le cocon (Eason et Whitcomb, 1965 ; Eberhard, 1974). Les jeunes de Lycosides se 
développeraient aussi bien en l'absence de leur mère qu'en sa présence (Higashi et Rovner, 1975). Mais les expériences ont été réalisées en laboratoire dans des conditions de températures stables et d'humidité élevée. Il n'en est certainement pas de même dans la nature. La plupart des Araignées onf une déperdition d'eau importante par évaporation. On peut donc considérer que la femelle de Lycosides favorise le développement de ses jeunes en effectuant une thermorégulation et en leur permettant d'accéder à l'eau.

Parfois, la nutrition des jeunes est indispensable à leur développement. Ceux d'Anelosimus studiosus sont incapables de se nourrir par eux-mêmes pendant la première semaine, même si on leur distribue de petites proies préalablement tuées (Brach, 1977). Chez Stegodyphus sarasinorum, le calamistrum et le cribellum permettant de tisser la soie cribellée adhésive nécessaire à la capture des proies, ne se développent qu'après la troisième ou quatrième mue. Les jeunes sont donc tributaires de la mère ou des autres individus de la colonie pour leur alimentation (Bradoo, 1972).

Les Araignées sont des animaux exclusivement prédateurs. Après la dispersion, qui survient le plus souvent avant la première prise alimentaire, les jeunes sont obligés de capturer les proies pour se nourrir. Ceci entraîne une mortalité importante, compensée par un taux de reproduction très élevé chez les espèces ne manifestant pas de comportement maternel. Le fait d'être nourris par la mère ne peuł que favoriser le développement des jeunes en leur permettant d'effectuer plusieurs mues avant de se disperser. Le prolongement de la vie grégaire des jeunes, après la mort de la mère, correspond à une adaptation supplémentaire. Ils unissent leurs efforts dans la capture des proies et bénéficient de ce fait d'un certain nombre d'avantages liés à la vie sociale (Krafft, 1979). Le comportement maternel des Araignées sociales n'est pas plus développé que celui des espèces solitaires, mais les chances de survie des jeunes sont augmentées par l'absence de discrimination que font les femelles entre leur progéniture et celle des congénères. La nutrition et la protection des jeunes ne sont donc plus liées à la survie de leur mère.

Il est souvent difficile d'évaluer objectivement l'aspect adaptatif d'un comportement, mais les Araignées pourraient constituer un modèle intéressant. Comme le nombre d'individus de chaque espèce est à peu près constant, nous pouvons considérer que le taux de reproduction est voisin du taux de mortalité. Celui-ci dépend, entre autres facteurs, du temps nécessaire au développement de l'individu et du degré d'adaptation de l'espèce à son milieu. Le nombre d'œufs pondus est généralement lié à la taille de l'espèce (Enders, 1976). Mais si nous comparons des espèces de taille à peu près voisine, nous pouvons constater que les Araignées dont le comportement maternel est très développé, ainsi que les espèces sociales, pondent très peu d'œufs. Le taux de reproduction semble inversement proportionnel aux soins dont peuvent bénéficier les jeunes. Parmi les Agelenides, Agelena labyrinthica pond entre 100 et 150 œufs par cocon. Coelotes terrestris entre 30 ef 70 ef Agelena consociata entre 10 et 20 . La même évolution s'observe chez les Theridiides, Theridion impressum (100 œufs), Theridion sisyphium (50), Anelosimus studiosus (35 à 50), Anelosimus eximius (20 d̀ 25) et chez les Eresides, Stegodyphus pacificus (450), Stegodyphus lineatus (100 à 120), Eresus niger (60), Stegodyphus sarasinorum (30 à 40), Stegodyphus mimosarum (20) (Brach, 1975. 1977 ; Krafft, 1970, 1979 ; Kullmann, 1970-71 ; Kullmann et Zimmermann, 1974). 


\title{
Mécanismes régulant le comportement maternel.
}

\author{
a) Soins aux cocons.
}

Le comportement maternel de la femelle dépend en partie de facteurs endogènes liés à son état physiologique. D'une façon générale il semble que la ponte soit un des facteurs déterminants de ce comportement. Si on offre un cocon à une femelle de Lycosides n'ayant pas encore pondu, elle le refuse ou le mange (Palmgern, 1944). Clubiona kulezynskii et Dolomedes plantarius font de même (Buckle, 1971 ; Bonnet 1940). En revanche, les femelles adultes de Theridion saxatile acceptent des cocons bien avant d'avoir elles-mêmes pondu des œufs (Norgaard, 1956).

Divers auteurs ont recherché les caractéristiques que devaient posséder les cocons pour être acceptés par une femelle manifestant un comportement maternel. Généralement celles-ci ne sont pas spécifiques. Theridion saxarile accepte les cocons de Theridion notatum (Norgaard, 1956). Pirata piraticus accroche un cocon de Pardosa à ses filières (Dieflein, 1967). Dolomedes plantarius transporte les cocons de Pisaura (Bonnet 1940). Stegodyphus lineatus soigne les cocons de Stegodyphus sarasinorium (Kullmann, 1972). On peut également faire accepter divers leurres par les Araignées telles que des boulettes de coton, de liège, de bois ou de plastique. Clubiona kulezynskii serait cependant plus exigeante. La structure superficielle du leurre joue le rôle le plus important. Son poids et sa composition chimique sont secondaires. Le cocon de Dolomedes plantarius pèse environ $0,5 \mathrm{~g}$, mais cette Araignée accepte des leurres 5 fois plus lourds. Fortement motivées, les Araignées se saisissent de pratiquement n'importe quoi et peuvent même transporter de petits cailloux (Lycosides) ou des proies en guise de cocon (Pisaurides). Ces diverses expériences suggèrent que les Araignées sont incapables de faire une discrimination entre un cocon et un leurre. Mais pour étudier la spécificité des stimulus en jeu, il est indispensable de proposer un choix aux Araignées, comme cela se pratique pour l'étude des phéromones de pistes, sexuelles ou sociales, contenues dans les fils de cheminement (Krafft et Roland, 1979). C'est uniquement par cette méthode que nous arriverons à déterminer la spécificité des stimulus.

Les travaux concernant l'aspect dynamique du comportement maternel des Araignées sont relativement rares. Certaines informations suggèrent cependant des mécanismes voisins de ceux mis en évidence par Vancassel (1977) sur les Dermaptères. Le comportement maternel des Araignées ne correspond pas à un enchaînement d'actes pré-programmés de manière rigide. Diverses informations provenant du cocon régulent ce comportement.

Agelena consociata fait une distinction entre des cocons contenant des œufs ou des larves ef des cocons morts ou vides. La stimulation que les cocons exercent sur les Araignées dépend également de leur position dans le nid (Krafft, 1970).

La persistance du comportement maternel dépend de la présence du cocon. Si l'on subtilise le cocon de Theridion sysiphium, celle-ci le refuse après un délai de $72 \mathrm{~h}$ (Locket, 1926). Les comportements maternels de Theridion impressum et de Theridion picfum disparaissent respectivement 3 jours $1 / 2$ et 6 jours après qu'on leur ait enlevé leur cocon (Hirschberg, 1969). II faut 5 jours de séparation chez Dolomedes plantarius (Bonnet, 1940). $20 \mathrm{~h}$ sont suffisantes pour Lycosa tarsalis (Palmgren, 1944). L'un des stimulus responsables de l'entretien de ce comportement maternel pourraît être chimi- 
que au moins chez Clubiona kulezynskii. Il disparaît si on lave les œufs à l'alcool (Buckle, 1971).

Il est également possible de prolonger le comportement maternel d'une Araignée en remplaçant son cocon par un cocon moins développé. En faisant accepter à Peucetia viridans un cocon de 12 jours plus jeune que le sien, on constate qu'elle l'ouvre 12 jours plus tard qu'elle n'aurait ouvert son propre cocon. Elle réagit certainement à un stimulus émanant du cocon au moment où les jeunes sont sur le point de sortir (Randall, 1977). Lycosa tarsalis fait de même (Palmgren, 1944). Les leurres n'émettant pas de signal adéquat, sont transportés plus longtemps que les cocons normaux par Dolomedes plantarius (Bonnet, 1940). L'ouverture du cocon par Pirata piraticus peut également être avancée, en lui substituant un cocon plus âgé. Cependant la substitution par un cocon plus jeune n'aurait pas d'effet (Dietlein, 1967).

La sortie des jeunes semble responsable de la disparition du comportement de soins au cocon. La disparition de ce comportement chez les Lycosides serait liée aux stimulations produites par les jeunes qui grimpent sur le dos de la femelle. Dix heures après le début de l'éclosion, lorsque les $2 / 3$ des jeunes sont sur le dos de la femelle, celle-ci refuse les cocons qu'on lui offre (Palmgren, 1944).

Le comportement prédateur des femelles est rarement inhibé pendant la période des soins au cocon. Clubiona kulezynskii n'absorbe pas de nourriture, mais cela semble lié au fait qu'elle reste enfermée dans sa logette. Si on lui offre des proies elle les capture. Coelotes terrestris, les Amaurobiides, les Theridiides et plusieurs Eresides manifestent un comportement prédateur normal et pourtant ne consomment pas leurs œufs. Il doit donc exister un stimulus, peut-être chimique, qui protège la ponte. Mais la femelle n'y est sensible que pendant la période où elle manifeste un comportement mafernel.

\section{b) Soins aux jeunes.}

Le comportement de soins aux jeunes dépend de mécanismes voisins de ceux qui régulent le comportement de soins au cocon. II est peu développé chez les Araignées errantes (Lycosides) et fait appel à des interactions complexes chez les Araignées sédentaires. Certaines de ces interactions sont comparables à celles qui existent chez les Araignées sociales.

Lorsque les jeunes Lycosides sortent du cocon, ils grimpent sur le dos de la femelle immobile. Ce rassemblement des jeunes sur le dos de la mère dépend essentiellement de stimulus tactiles liés à la structure tégumentaire (Dietlein, 1967 ; Higashi et Rovner, 1975 ; Job, 1974). L'abdomen des Lycosides femelles adultes est garni de poils se terminant en boutons qui permettent aux jeunes de s'accrocher. Ces poils, dépourvus d'innervation, sont absents chez les mâles adultes et les femelles subadultes (Rovner, Higashi ef Foelix 1973, Job, 1974). Ce stimulus tactile n'est pas spécifique car les jeunes acceptent de grimper sur le dos de femelles d'autres espèces de Lycosides à condition qu'elles soient dans l'état psychophysiologique requis et qu'elles restent immobiles. Les jeunes seraient à leur tour à l'origine de stimulations au niveau des poils mécanorécepteurs de la femelle, entretenant son comportement maternel (Rovner, Higashi et Foelix, 1973). La durée du comportement de soins aux jeunes est en effet variable. Aulonia albimana (Lycosidae) transporte normalement ses jeunes pendant 4 à 5 jours, 
mais ce comportement peut se prolonger jusqu'à 21 jours lorsque la température ambiante est basse (Job, 1974).

Les interactions sont plus complexes lorsque le comportement maternel exige une coordination étroite entre les activités des individus. Les jeunes Lycosides tissent un fil de cheminement en descendant du dos de la femelle pour s'abreuver. Dès que la femelle esquisse un mouvement, les variations de tension de ces fils incitent les jeunes à remonter sur la mère (Higashi et Rovner, 1975). Theridion saxatile nourrit ses jeunes en leur distribuant des proies pendant un mois. Pendant les premières semaines elle tiraille les fils de ta toile de manière saccadée lorsqu'elle capture une proie. Ces signaux vibratoires ont pour effet de faire refluer tous les jeunes vers l'abri. Quand la proie est paralysée, la femelle exerce des tractions lentes sur les fils, ce qui attire les jeunes (Norgaard, 1956 ; Hirschberg, 1969). Theridion pictum utiliserait également un signal vibratoire pour attirer les jeunes. La femelle de Coelotes farrestris fait de même (Tretzel, 1961). Mais il s'agit d'observations qui nécessitent une vérification en enregistrant puis en restituant ces vibrations. La nutrition des jeunes par régurgitation exige une stimulation des palpes et de la région buccale de la femelle. Cet échange trophallactique peut être interrompu à tous moments par la femelle qui écarte les jeunes à l'aide de ses pattes (Hirschberg, 1969).

La cohésion du groupe de jeunes n'est pas nécessairement liée à la présence de la mère, car leur vie grégaire peut se poursuivre après la mort de celle-ci. II existe une tendance au groupement chez les Lycosides (Dietlein, 1967 ; Fujii, 1976). Mais chez les espèces sédentaires la cohésion du groupe dépend également de l'attraction exercée par les structures soyeuses élaborées par la femelle et complétées par les jeunes. Par la suite, le développement de l'agressivité des jeunes entraînerait la dispersion des individus (Krafft, 1979). Les mécanismes responsables de la cohésion du groupe n'ont été étudiés jusqu'à présent que chez les Araignées sociales.

Le comportement prédateur €st rarement supprimé chez les femelles qui soignent leurs jeunes. II est donc indispensable que ceux-ci soient protégés des attaques de la femelle. Selon certains auteurs, la femelle ne serait pas capable de détecter la présence de ses jeunes. Job (1974) explique la tolérance dont bénéficient les jeunes par une élévation du seuil de déclenchement du comportement prédateur d'Aulonia albimana. Cyrtophora moluccencis tolère tous les jeunes dont la longueur est inférieure à $8 \mathrm{~mm}$ (Lubin, 1974). Les ébranlements de la toile provoqués par les jeunes seraient trop faibles pour déclencher une réponse de la part de la femelle. En fait, cette explication est insuffisante pour les cas où des relations précises doivent s'établir entre la mère et les jeunes, notamment chez les espèces où la progéniture est nourrie par la mère ou bien participe avec elle à la capture des proies. Theridion impressum réagit aux mouvements de ses jeunes en tiraillant les fils de la toile. Le comportement maternel de Coelofes ferrestris peut être prolongé au laboratoire sans que la mère ne manifeste d'agressivité à l'égard de ses jeunes, malgré leur taille devenue importante (Horel, Roland ef Leborgne, 1979). Il est alors nécessaire d'envisager une reconnaissance de la forme des signaux vibratoires provoqués par un jeune qui se déplace sur la toile (Krafft et Leborgne, 1979 ; Leborgne et Krafft, 1979) c'est dans ce sens que vont les travaux de Tretzel (1961) chez Coelotes terrestris. Les signaux doivent être voisins pour toutes les espèces, de taille similaire, appartenant à un même genre ou à une même famille. Ainsi, on peut échanger des jeunes de Theridion pictum et Th. impressum (Hirschberg, 
1969), de Th. impressum et Th. sisyphium, de Stegodyphus lineatus ef St. sarasinorum (Kullmann, 1972). Il en est de même chez plusieurs Lycosides (Meyer, 1928). En revanche Theridion impressum capture des jeunes d'Araneus, de Pirata piraticus et même de Theridion pictum si ceux-ci sont trop grands. Mais une reconnaissance à distance n'est pas seule à intervenir. Le comportement maternel implique des contacts directs qui font intervenir des stimulus complémentaires inhibant le cannibalisme comme chez les Araignées sociales (Krafft, 1975). Chez les espèces non sociales ef subsociales, cette tolérance s'exerce exclusivement à l'égard des jeunes. Les femelles sont donc capables de faire une discrimination entre jeunes et adultes en fonction d'un stimulus propre aux jeunes.

La tolérance de la mère à l'égard des jeunes dépend également de son état physiologique. Les femelles de Cyrtophora citricola ne tolèrent les jeunes que si elles ont déjà elles-mêmes confectionné un cocon (Blanke, 1972). Les Lycosides non fécondées ou subadultes n'acceptent pas les jeunes.

La mère doit également être protégée des attaques de ses jeunes puisque leur comportement prédateur est développé. Cette tolérance doit dépendre d'un mécanisme qui se rapproche de la tolérance réciproque des espèces sociales. En effet, les jeunes se tolèrent entre eux et tolèrent tout adulte, qu'il s'agisse de leur mère ou d'une autre femelle. L'inhibition du cannibalisme chez les jeunes dépendrait donc d'un stimulus présent chez tous les individus de l'espèce quel que soit leur âge. La disparition de cefte tolérance au moment de la dispersion peut alors s'expliquer par une baisse de réactivité des jeunes à ce stimulus. L'environnement est susceptible, au moins dans certains cas, d'agir sur l'évolution de la réactivité, comme le montre la possibilité de prolonger expérimentalement la phase grégaire des jeunes de Coelofes terrestris jusqu'à l'âge subadulte (Horel, Roland et Leborgne, 1979).

Le comportement maternel ferait donc appel à deux mécanismes de tolérance distincts, l'un lié à l'état psychophysiologique de la femelle et dépendant d'un stimulus spécifique aux jeunes, l'autre protégeant les jeunes entre eux et la mère des jeunes, lié à l'état psychophysiologique des jeunes et dépendant d'un stimulus de type social répandu chez tous les individus.

\section{Conclusion.}

Le comportement maternel des Araignées peut se limiter à l'abandon du cocon dans un endroit favorable au développement des œufs ou s'étendre jusqu'à une protection et une nutrition des jeunes. Son apparition est liée à la ponte, mais divers mécanismes de régulation permettent l'ajustement du comportement de la femelle. Le cocon est à l'origine de stimulus qui entretiennent le comportement maternel. Celui-ci peut être écourté ou prolongé. Le comportement de soins aux jeunes dépend des stimulations que ceux-ci exercent sur la femelle. De nombreuses espèces nourrissent leurs jeunes en leur distribuant des proies préalablement injectées de sucs digestifs. Certains Theridiides et Eresides nourrissent leur progéniture par régurgitation à la suite de mouvements de sollicitations par les jeunes. Lorsque les jeunes coopèrent dans la capture des proies, ils forment une structure de groupe voisine de l'organisation sociale. La persistance de cette association, même en l'absence de la mère, est certainement une voie qui a conduit à l'apparition des sociétés d'Araignées. 
Les interactions entre la mère et les jeunes font intervenir des mécanismes de tolérance indispensables chez ces animaux exclusivement prédateurs. La mère distingue les ébranlements de la toile provoqués par une proie de ceux produits par les jeunes qui se déplacent. Les jeunes se tolèrent entre eux, ainsi que leur mère. Cette reconnaissance, fondée en grande partie sur l'utilisation d'informations vibratoires, est liée à l'existence de structures soyeuses dont le rôle est considérable chez la plupart des espèces (Witt, 1975). Le comportement maternel correspond sans aucun doute à un caractère adaptatif car le taux de reproduction de ces espèces est considérablement diminué traduisant une réduction de la mortalité.

Les Araignées constituent, à plusieurs titres, un modèle intéressant pour l'étude du comportement maternel. L'acceptation ef le transport du cocon sont des critères pratiques pour aborder son aspect ontogénétique. La tolérance réciproque entre mère et jeunes met en causes des facteurs physiologiques et environnementaux. II est relativement aisé de manipuler les signaux vibratoires transmis par la toile et intervenant dans les relations interindividuelles.

Colloque « La relation parentale » Nouzilly, France, juin 1979.

\section{Références}

BLANKE R., 1972. Untersuchungen zur Okophysiologie und Okethologie von Cyrfophora citricola Forskal (Araneae, Araneidae) in Andalusien. Forma ef functio, 5, 125-206.

BONNET P., 1940. L'instinct maternel des Araignées à l'épreuve de l'expérimentation. Bull. Soc. Hist. nat. Toulouse, 81, 185-250.

BRACH V., 1975. The biology of the social spider. Anelosimus eximius (Araneae Theridiidae). Bull Soc. Calif. Acad. Sci., 74, 37-41.

BRACH V., 1976. Subsocial behavior in the funnel-web Wolf spider Sosippus floridanus (Araneae : Lycosidae). Florida Entomol., 59, 225-229.

BRACH V., 1977. Anelosimus studiosus (Araneae: Theridiidae) and the evolution of quasisociality in Theridiid spiders. Evolution, 31, 154-161.

BRADOO B. L. 1972. Some observations on the ecology of social spider Stegodyphus sarasinorum Karsh (Araneae, Eresidae) from India. Orient. Insects, 6, 193-203.

BRISTOWE W. S., 1958. The World of Spiders, pp. 304. Collins, London.

BUCHLI H., 1960. Quelques observations concernant le cycle de développement chez la Mygale maçonne Nemesia caementaria Latr. C. R. Acad. Sc., Paris, 251, 2773-2775.

BUCHLI H., 1968. Notes sur la Mygale terricole Cteniza moggridgei (Pick. Cambr. 1874). Rev. Ecol. Biol. Socl. T. 5, 1-40.

BUCKLE D. J., 1971. Social behavior in Spiders with special reference to maternal behavior in Clubiona kulezynski (Lessert). Proceed. 19th Annu. Meet. Enfomol. Soc. Saskatchewan.

CLOUDSLEY-THOMPSON J. L., 1955. The life histories of the British cribellate spiders of the genus Ciniflo BI. (Dictynidae). Ann. Mag. Natur. Hist., 12, 787-794.

DIETLEIN W., 1967. Ein Beitrag zur Sexualbiologie und Brutfürsorge bei Lycosiden, mit besonderer Berücksichtigung von Pirata piraticus (Clerk), Thèse Nurnberg.

EASON R., WHITCOMB W. H., 1965. Life history of the dotted Wolf Spider Lycasa punctulafa H. (Araneida ; Lycosidae). Arkansos Acad. Sci. Proceed., 19, 11-20.

EASON R. R., PECK W. B., WHITCOMB W. H., 1967. Notes on spider parasites, including a reference list. J. Kansas Entomol. Soc., 40, $422-434$.

EBERHARD W. G., 1974. Maternal behaviour in a South American Lyssomanes. Bull. brit. Arachn. Soc., $3,51$.

ENDERS F., 1976. Clutch size related to hunting manner of Spider species. Ann. Entomol. Soc. Am., 69, 991-998. 
FUJII Y., 1976. Pulli-carrying Behaviour in Wolf Spiders (Lycosidae Araneae). Bull. rippon dental Univ. Gen. Educ., 5, 145-151.

FUJll Y., 1978. Examinations of the Maternal Care of cocon in Pardosa astrigera L. Koch. (Aranease, Lycosidae). Bull. nippon dental Univ., Gen. Educ., 7, 223-230.

HIGASHI G. A., ROVNER J. S., 1975. Post-emergent behaviour of juvenile lycosid spiders. Bull. brit. Arachn. Soc., 3, 113-119.

HITE J. M., GLADNEY W. J., LANCASTER J. L., WHITCOMB W. J., 1966. Biology of the Brown Recluse Spider. Arkansas exper. Stat. Bull., no 711. 1-26.

HIRSCHBERG D., 1969. Beiłräge zur Biologie, insbesondere zur Brutpflege einiger Theridiiden. Z. wiss. Zool., 179, 189-252.

HOREL A., ROLAND Ch., LEBORGNE R., 1979. Mise en évidence d'une tendance au groupement chez les jeunes de l'Araignée solitaire Coelotes terrestris. Rev., arachnol., 2, 157-164.

HUMPHREYS W. F., 1974. Behavioural thermoregulation in a wolf spider. Nature, 251, $502-503$.

JACSON C. C., JOSEPH K. J., 1973. Life history, bionomics and behaviour of the social spider Stegodyphus sarasinorum Karsch. Insectes soc., 20, 189-204.

JOB W., 1974. Beiträge zur Biologie der fangnetzbauenden Wolfsspinne Aulonia albimana (Walck 1805). Zool. jb. Syst., 101, 560-608.

KESSLER A., FOKKINGA A., 1973. Hymenopterous parasites in egg sacs of Spiders of the genus Pardosa (Araneidae Lycosidae). Tijdschr. Entomol., 116, 43-61.

KRAFFT B., 1970. Contribution à la biologie et à l'éthologie d'Agelena consociata Denis (Araignée sociale du Gabon). I. Biol. gabon., 3, 197-301.

KRAFFT B., 1975. La tolérance réciproque chez l'Araignée sociale Agelena consociafa Denis. Proc. 6th int. Arachn. Congr., Amsterdam, 107-112.

KRAFFT B., 1979. Organisation et évolution des sociétés d'Araignées. J. Psychol., 1, $23-51$.

KRAFFT B., LEBORGNE R., 1979. Perception sensorielle et importance des phénomènes vibratoires chez les Araignées. J. Psychol. (sous-presse).

KRAFFT B., ROLAND Ch., 1979. Un labyrinthe appliqué à l'étude des attractions sociale et sexuelle et de leur spécificité chez les Araignées. Rev. arachnol., 2, 165-171.

KULLMANN E., 1970-71. Bemerkenswerte Konvergenzen im Verhalten cribellater und ecribellater Spinnen. Freunde des Kölner Zoo, 4, 123-150.

KULLMANN E., 1972. Evolution of social behavior in Spiders (Araneae; Eresidae and Theridiidae). Am. Zool., 12, 419-426.

KULLMANN E., NAWAB! St., ZIMMERMANN W., 1972. Neue Ergebnisse zur Brutbiologie cribellater Spinnen aus Afghanistan und der Serengeti. Z. Kölner, Zoo, 3, 87-108.

KULLMANN E., SITTERTZ H., ZIMMERMANN W., 1971. Erster Nachweis von Regurgitationsfütterungen bei einer cribellaten Spinne (Stegodyphus lineatus Latreille, 1817, Eresidae). Bonn. Zool. Beitr., 22, 175-188.

KULLMANN E., ZIMMERMANN W., 1974. Regurgitationsfütterungen als Bestandteil der Brutfürdorge bei Haubennetz und Röhrenspinnen (Araneae, Theridiidae und Eresidae). Proc. 6th int. Arachn. Congr. Amsterdam, 120-124.

LEBORGNE R., KRAFFT B. 1979. Techniques d'enregistrement et d'analyse des signaux vibratoires intervenant dans les comportements des Araignées sédentaires. Rev. Arachnol. 2, 173-182.

LE PAPE G., 1972. Contribution à l'étude du comportement reproducteur en liaison avec l'alimentation chez quatre arachnides : Teutana grossa, Pardosa lugubris, Pisaura mirabilis, Buthus occitanus. Th. 3e Cycle, Rennes.

LOCKET G. H., 1926. Observations on the mating habits of some web-spinning Spiders. Proc. Zool. Soc. London, 74, 1125-1146.

LUBIN Y. D., 1974. Adaptative advantages and the evolution of colony formation in Cyrtophoro (Araneae, Araneidae). Zool. J. Linn. Soc., 54, 321-339.

MEYER E., 1928. Neue Sinnesbiologische Beabachfungen an Spinnen. Z. Morphol. Okol. Tiere, 12, 1-69.

NORGAARD E., 1956. Environment and behaviour of Theridion saxatile. Oikos 7, 159-192.

PALMGREN P., 1944. Uber die Brutpflegeinstinkthandlungen der Wolfspinnen (Lycosidae). Soc. Sci. Fenn., Comment. biol., 9, 1-29.

RANDALL J. B., 1977. New observations of maternal care exhibited by the green Lynx Spider Peucetio viridans Heutz (Araneae, Oxyopidae). Psyche, 84, 286-291. 
ROVNER J. S., HIGASHI G. A., FOELIX R. F., 1973. Maternal behavior in Wolf Spiders : The role of abdominal hairs. Science, 182, 1153-1155.

SHEAR W. A., 1970. The evolution of social phenomena in spiders. Bull. brit. Arachn. Soc., 1, 65-76.

TRETZEL E., 1961. Biologie, Okologie und Brutpflege von Coelotes terrestris (Wider), (Araneae, Agelenidae). Z, Morphol. Okol. Tiere, 50, 375-542.

TRETZEL E., 1963. Die Sprache bei Spinnen. Umsch., 13, 403-407.

VANCASSEL M., 1977. Le développement du cycle parental de Labidura riparia. Biol. Comport., 2, 5175.

WICKLER W., 1973. Uber Koloniegründung und soziale Bindung von Stegodyphus mimosarum Pavesi und anderen sozialen Spinnen. Z. Tierpsychol., 32, 522-531.

WITT P. N., 1975. The web as a means of communication. Biosc. Commun., 1, 7-23. 